\title{
Direct URCA-processes in neutron star quark core with strong magnetic field.
}

\author{
Vasily Belyaev ${ }^{1, \star}$ and Alexander Gvozdev ${ }^{1, \star \star}$ \\ ${ }^{1}$ Yaroslavl State University, Russia, Yaroslavl, Sovyetskaya st., 21
}

\begin{abstract}
Neutrino emissivity in direct URCA processes in superdense strongly magnetized quark medium is calculated. In calculations, we use the covariant method of density matrixes for charge fermions in uniform constant magnetic field. One-gluon exchanges between quarks are also take into account.

In evaluations, the strength of magnetic field corresponds to the case, where the quarks of medium occupy a lot of Landau levels, while the electrons are in ground Landau level. The analytical dependence of neutrino emissivity on chemical potentials of quarks and electrons, temperature and magnetic field strength is obtained and briefly discussed. The result could be important in application to a massive strongly magnetized neutron star with quark core.
\end{abstract}

\section{Introduction}

Discovery of neutron stars in the $1.9-2.0 M_{\text {sun }}$ mass range [1] opened the wide discussion of the matter structure in the inner core of such a massive neutron star. In particular, model of neutron star core, consisting of strange quark matter, is discuss and develop at present time [2]. Neutrino cooling of neutron star core is very important, because of such a cooling is responsible for determination and calculation theoretically of neutron star cooling curve [3].

On the basis on observations made to date, there were discovered the group of neutron stars with strong magnetic fields, so called magnetars [4] with magnetic induction $B$ up to $10^{15} G$ on the surface.

Hence, it is logically to investigate the neutrino cooling rate of a neutron star with strongly magnetized quark core. Without magnetic field, such a work was done and published in 1980 [5]. In this work cold electroneutral medium in beta-equilibrium consists of ideal gases of u-, d-, s-quarks and electrons was considered. It was shown, that it is necessary to include s-quark, because in that case the parameter "bound energy per quark" must be minimal [6].

To describe week interaction, we use Lagrangian of four-fermion interaction:

$$
\mathcal{L}=\frac{G_{F} \cos \theta_{c}}{\sqrt{2}}\left[\bar{d} \gamma_{\alpha}\left(g_{v}+g_{a} \gamma^{5}\right) u\right]\left[\bar{v} \gamma^{\alpha}\left(1+\gamma^{5}\right) e\right]
$$

where $G_{F}$ is Fermi constant, $\theta_{c}$ is Cabbibo angle, $g_{v}, g_{a}$ are vector and axial electroweak constants. We assume, that in the limit of cold quark matter the constants depend on chemical potentials of quarks

\footnotetext{
$\star^{\star}$ e-mail: SCstalk@mail.ru

${ }^{\star}$ e-mail: gvozdev@uniyar.ac.ru
} 
only, and the following URCA-processes give leading contribution in neutrino emissivity:

$$
\begin{gathered}
u+e^{-} \rightarrow d+v_{e}, \quad \text { (crossing) } \\
d \rightarrow u+e^{-}+\bar{v}_{e} . \quad \text { (decay) }
\end{gathered}
$$

One neglects contributions in neutrino emissivity of similar URCA-processes with $s$-quark, because they are suppressed by Cabbibo angle. If one takes into account one-gluon exchanges between quarks, then the neutrino emissivity is not negligible and proportional to $G_{F}^{2} \alpha_{s} \mu_{u} \mu_{d} \mu_{e} T^{6}$ [5]. Here $\alpha_{s}$ is strong coupling constant, $\mu_{u}, \mu_{d}, \mu_{e}$ are chemical potentials of $u$-, $d$-quarks and electrons accordingly, $T$ is temperature. This is well-known old result, cited in many reports and books on neutron stars.

In the present paper we consider the quark core of massive neutron star with strong magnetic field. We assume, that:

$$
\mu_{u}^{2}, \mu_{d}^{2} \gg m_{q}^{2}(\mu)>e B \gtrsim \mu_{e}^{2} \gg T^{2}
$$

where

$$
m_{q}^{2}(\mu)=\frac{2 \alpha_{s}}{3 \pi} \mu_{q}^{2}
$$

is well-known effective quark mass [7] in the one-gluon approximation of finite-temperature QCD in the limit $T \rightarrow 0$, B is the magnetic field strength. The first two inequations in (4) means, that magnetic field has small effect on QCD-dynamics, consequently, on effective quark masses and a lot of Landau levels are occupied by quarks. The third inequation mean, that electrons are occupied ground Landau level only. Substitute in inequation (4) reasonable values of chemical potentials quarks and electrons $\mu_{u, d} \approx 500 \mathrm{MeV}, \mu_{e} \approx 30 \mathrm{MeV}$, one obtain:

$$
B \sim 10^{17} \mathrm{G} .
$$

We note, that this value of magnetic field is reasonable for neutron star core and is not effect substantially on equation of state of quark medium. We assume, that the quarks are ideal massive gas with mass $m(\mu)$, while electrons and neutrino are massless.

For evaluation neutrino emissivity we use matrix density technics for charged fermion in external magnetic field [8]. The conservation laws of 4-momentum for crossing and decay are:

$$
\mathcal{P}_{1}+p=\mathcal{P}_{2}+k, \quad \mathcal{P}_{2}=\mathcal{P}_{1}+p+k,
$$

where $\mathcal{P}_{1}, \mathcal{P}_{2}, p, k-4$-momenta of u-, d-quarks, electron and neutrino accordingly,

$$
\mathcal{P}_{1}^{\mu}=\left(E_{n}, \overrightarrow{\mathcal{P}}_{1}\right), \quad \mathcal{P}_{2}^{\mu}=\left(E_{n^{\prime}}, \overrightarrow{\mathcal{P}}_{2}\right), \quad p^{\mu}=\left(\epsilon_{n^{\prime \prime}}, \vec{p}\right), \quad k^{\mu}=(\omega, \vec{k}),
$$

where $E_{n}=\sqrt{p_{z}^{2}+2 q e B n+m^{2}}$ is the energy of charged particle in external magnetic field, $q$ is module of particles charge, $\epsilon_{n^{\prime \prime}}=\epsilon_{0} \equiv \epsilon$, because all electrons take ground Landau level.

Neutrino emissivities of decay and crossing are presented as follows:

$$
\begin{gathered}
Q^{(\mathrm{dec})}=\frac{3}{V} \sum d n_{d} f_{d} \sum d n_{u}\left(1-f_{u}\right) \sum d n_{e}\left(1-f_{e}\right) \sum d n_{v} \omega \frac{\left|S_{i f}^{(\mathrm{dec})}\right|^{2}}{\tau}, \\
Q^{(\mathrm{cr})}=\frac{3}{V} \sum d n_{u} f_{u} \sum d n_{e} f_{e} \sum d n_{d}\left(1-f_{d}\right) \sum d n_{v} \omega \frac{\left|S_{i f}^{(\mathrm{cr})}\right|^{2}}{\tau} .
\end{gathered}
$$

where sums mean summing under overall phase volumes of particles, $f_{i}$ are the distributions functions of particles, $\omega$ is neutrino energy, $V$ is normalized volume, $\tau$ is normalized time, $\left|S_{i f}\right|^{2}$ is squared 
S-matrix element. It is assumed in formulae, that emitting medium is transparent for neutrinos. The multiplier factors " 3 " take into account, because the quarks with all colours participate in all processes, and the colour conservates in processes.

We discuss below the crossing process, because the evaluations are similar for both processes. After simplifications, the neutrino emissivity of crossing $Q^{\text {(cr) }}$ could be present in the form:

$$
\begin{aligned}
& Q^{(\mathrm{cr})}=\frac{3 G_{F}^{2} \cos ^{2} \theta_{c} g_{v}^{2}}{32(2 \pi)^{8}} \sum_{n, n^{\prime}} g_{n} g_{n^{\prime}} \int \frac{d^{3} \mathcal{P}_{1}}{E_{n}} f_{u} \int \frac{d^{3} p}{\epsilon} f_{e} \cdot \\
& \cdot \int \frac{d^{3} \mathcal{P}_{2}}{E_{n^{\prime}}}\left(1-f_{d}\right) \int d^{3} k \delta^{4}\left(\mathcal{P}_{1}+p-\mathcal{P}_{2}-k\right) S p\left[\hat{\rho}_{d} \gamma_{\alpha} .\right. \\
&\left.\cdot\left(1+c \gamma^{5}\right) \hat{\rho}_{u} \gamma_{\beta}\left(1+c \gamma^{5}\right)\right] S p\left[\hat{\rho}_{\nu} \gamma^{\alpha}\left(1+\gamma^{5}\right) \hat{\rho}_{e} \gamma^{\beta}\left(1+\gamma^{5}\right)\right],
\end{aligned}
$$

where $c=g_{a} / g_{v}, \hat{\rho}_{u}, \hat{\rho}_{d}, \hat{\rho}_{e}, \hat{\rho}_{v}$ - are density matrices of u-, d-quarks, electron, summed over spins, and neutrino accordingly, $g_{n}$ is the factor of spins degeneration.

We take the density matrices for $\mathrm{u}, \mathrm{d}$, e from [8], and don 't forget, that the quarks have fractional charges. One evaluates the traces by standard rules [8]. After long and tedious evaluations, we obtain:

$$
\begin{gathered}
Q^{(\mathrm{cr})}=\frac{6 G_{F}^{2} \cos ^{2} \theta_{c} g_{v}^{2}}{(2 \pi)^{8}} \sum_{n, n^{\prime}} g_{n} g_{n^{\prime}} \int \frac{d^{3} \mathcal{P}_{1}}{E_{n}} f_{u} \int \frac{d^{3} p}{\epsilon} f_{e} \cdot \\
\cdot \int \frac{d^{3} \mathcal{P}_{2}}{E_{n^{\prime}}}\left(1-f_{d}\right) \int d^{3} k \delta^{4}\left(\mathcal{P}_{1}+p-\mathcal{P}_{2}-k\right)(-1)^{n+n^{\prime}} \exp \left(-\frac{u_{u}+u_{d}+u_{e}}{2}\right) \cdot \\
\cdot\left(\left[(1+c)^{2} L_{n^{\prime}-1}\left(u_{d}\right) L_{n}\left(u_{u}\right)+(1-c)^{2} L_{n-1}\left(u_{u}\right) L_{n^{\prime}}\left(u_{d}\right)\right] \Phi_{\|}+8(1+c)^{2} L_{n^{\prime}-1}^{1}\left(u_{d}\right) L_{n}\left(u_{u}\right)\left(\mathcal{P}_{2} \Lambda k\right)\left(\mathcal{P}_{1}(\tilde{\Lambda}+\tilde{\phi}) p\right)+\right. \\
+8(1-c)^{2} L_{n-1}^{1}\left(u_{u}\right) L_{n^{\prime}}\left(u_{d}\right)\left(\mathcal{P}_{1} \Lambda k\right)\left(\mathcal{P}_{2}(\tilde{\Lambda}+\tilde{\phi}) p\right)+ \\
\left.+4 L_{n^{\prime}}\left(u_{d}\right) L_{n}\left(u_{u}\right)(k(\tilde{\Lambda}+\tilde{\phi}) p)\left[\left(1+c^{2}\right)\left(\mathcal{P}_{2} \tilde{\Lambda} \mathcal{P}_{1}\right)-\left(1-c^{2}\right) m_{u} m_{d}+2 c\left(\mathcal{P}_{1} \tilde{\phi} \mathcal{P}_{2}\right)\right]\right), \quad(12)
\end{gathered}
$$

where

$$
\begin{aligned}
\Phi_{\|} \equiv 2\left(\mathcal{P}_{2} \tilde{\Lambda} \mathcal{P}_{1}\right)(k \tilde{\Lambda} p) & -2\left(\mathcal{P}_{2} \tilde{\Lambda} p\right)\left(\mathcal{P}_{1} \tilde{\Lambda} k\right)-2\left(\mathcal{P}_{2} \tilde{\Lambda} k\right)\left(\mathcal{P}_{1} \tilde{\Lambda} p\right)- \\
& -\left(\mathcal{P}_{2} \tilde{\Lambda} k\right)\left(\mathcal{P}_{1} \tilde{\phi} p\right)-\left(\mathcal{P}_{1} \tilde{\Lambda} k\right)\left(\mathcal{P}_{2} \tilde{\phi} p\right)-\left(\mathcal{P}_{1} \tilde{\Lambda} p\right)\left(\mathcal{P}_{2} \tilde{\phi} k\right)-\left(\mathcal{P}_{2} \tilde{\Lambda} p\right)\left(\mathcal{P}_{1} \tilde{\phi} k\right) .
\end{aligned}
$$

Here $\phi_{\mu v}=F_{\mu \nu} / B, \tilde{\phi}_{\mu v}=\tilde{F}_{\mu v} / B$ are dimensionless tensor, dual tensor of magnetic field, $\Lambda_{\mu v}=(\phi \phi)_{\mu \nu}$, $\tilde{\Lambda}_{\mu v}=(\tilde{\phi} \tilde{\phi})_{\mu \nu}, L_{n}^{k}$ are Laguerre polynomials, $u_{d}=6 \mathcal{P}_{2 \perp}^{2} / e B, u_{u}=3 \mathcal{P}_{1 \perp}^{2} / e B, u_{e}=2 p_{\perp}^{2} / e B, p_{\perp}^{2}$ is denoted the transversal to magnetic field strength components of charge particles momenta, for example, electron squared.

\section{Integrals on transversal to magnetic field strength components of charge particles momenta}

To calculate the neutrino emissivity (12), it is necessary to evaluate the following scalar and vector integrals:

$$
\begin{aligned}
\mathcal{J}^{(n, m)}=\int d \mathcal{P}_{1 x} d \mathcal{P}_{1 y} \int d \mathcal{P}_{2 x} d \mathcal{P}_{2 y} \int d p_{x} d p_{y} L_{n}\left(u_{u}\right) L_{m}\left(u_{d}\right) & \\
& \exp \left[-\frac{u_{u}+u_{d}+u_{e}}{2}\right] \delta_{\perp}\left(\mathcal{P}_{1}+p-\mathcal{P}_{2}-k\right),
\end{aligned}
$$




$$
\begin{aligned}
\mathcal{J}^{(n, m) \alpha}=\int d \mathcal{P}_{1 x} d \mathcal{P}_{1 y} \int d \mathcal{P}_{2 x} d \mathcal{P}_{2 y} \int d p_{x} d p_{y} \mathcal{P}_{1}^{\alpha} L_{n-1}^{1}\left(u_{u}\right) L_{m}\left(u_{d}\right) \cdot \\
\exp \left[-\frac{u_{u}+u_{d}+u_{e}}{2}\right] \delta_{\perp}\left(\mathcal{P}_{1}+p-\mathcal{P}_{2}-k\right), \\
\tilde{\mathcal{J}}^{(n, m) \alpha}=\int d \mathcal{P}_{1 x} d \mathcal{P}_{1 y} \int d \mathcal{P}_{2 x} d \mathcal{P}_{2 y} \int d p_{x} d p_{y} \mathcal{P}_{2}^{\alpha} L_{n}\left(u_{u}\right) L_{m-1}^{1}\left(u_{d}\right) . \\
\exp \left[-\frac{u_{u}+u_{d}+u_{e}}{2}\right] \delta_{\perp}\left(\mathcal{P}_{1}+p-\mathcal{P}_{2}-k\right),
\end{aligned}
$$

where $\delta_{\perp}\left(\mathcal{P}_{1}+p-\mathcal{P}_{2}-k\right)=\delta\left(\mathcal{P}_{1 x}+p_{x}-\mathcal{P}_{2 x}-k_{x}\right) \delta\left(\mathcal{P}_{1 y}+p_{y}-\mathcal{P}_{2 y}-k_{y}\right)$. We have calculated these integrals similarly to [8], and obtained:

$$
\begin{gathered}
\mathcal{J}^{(n, m)}(v)=\frac{\pi^{2}}{3}(e B)^{2}(-1)^{n+m} I_{0}^{(n, m)}(v), \\
\mathcal{J}^{(n, m) \alpha}(v)=k_{\perp}^{\alpha} \frac{\pi^{2}}{6}(e B)^{2}(-1)^{n+m+1} n\left(I_{1}^{(n-1, m)}(v)-I_{1}^{(n, m)}(v)\right), \\
\tilde{\mathcal{J}}^{(n, m) \alpha}(v)=k_{\perp}^{\alpha} \frac{\pi^{2}}{6}(e B)^{2}(-1)^{n+m} m\left(I_{1}^{(n, m-1)}(v)-I_{1}^{(n, m)}(v)\right) .
\end{gathered}
$$

Here $v^{2}=k_{\perp}^{2} / e B, k_{\perp}^{2}=\left(k_{x}\right)^{2}+\left(k_{y}\right)^{2}$ is the neutrino transverse momenta squared and integrals $I_{i}^{n, m}$ are determined, as follows:

$$
\begin{gathered}
I_{0}^{(n, m)}(v) \equiv \int_{0}^{\infty} d x e^{-3 x} L_{n}(2 x) L_{m}(x) J_{0}(\sqrt{6} v \sqrt{x}), \\
I_{1}^{(n, m)}(v) \equiv \sqrt{\frac{2}{3}} \frac{1}{v} \int_{0}^{\infty} d x \frac{e^{-3 x}}{\sqrt{x}} L_{n}(2 x) L_{m}(x) J_{1}(\sqrt{6} v \sqrt{x}),
\end{gathered}
$$

where $J_{0}, J_{1}$ - are Bessel functions. The functions $I_{0}^{(n, m)}(v), I_{1}^{(n, m)}(v)$ at fixed values of $v$ have asymmetric delta-like "burst" behavior at $\mathbf{m}=\mathbf{2 n}-\mathbf{1}$. If values $n, m$ turn from tens to thousands, then the "burst" diffuses fast.

From this point we use various approximations. First, we assume, that the parameter $v^{2}=k_{\perp}^{2} / e B$ is small $(v \ll 1)$. Hence, one evaluate approximately functions $I_{0}, I_{1}$. One approximate $J_{1}(z) \approx z / 2$, $J_{0}(z) \approx 1$, after that the leading terms in expansion series on $v$ are:

$$
I_{1}^{(n, m)}(v)=I_{0}^{(n, m)}(v) \approx \int_{0}^{\infty} d x e^{-3 x} L_{n}(2 x) L_{m}(x) \equiv C_{0}^{(n, m)} .
$$

One can evaluate the function $C_{0}^{(n, m)}$ exactly [9]

$$
C_{0}^{(n, m)}=\frac{\Gamma(n+m+1)}{n ! m !} \frac{2^{m}}{3^{n+m+1}} .
$$

Second, we assume, that the leading contribution in neutrino emissivity gives the region $n, n^{\prime} \gg 1$. Hence, we replace

$$
g_{n}=g_{n^{\prime}}=2 .
$$

Moreover, where it does not cause confusion, we replace

$$
(n-1) \rightarrow n, \quad\left(n^{\prime}-1\right) \rightarrow n^{\prime} .
$$


In expressions like $\left(I_{1}^{\left(n, n^{\prime}-1\right)}-I_{1}^{\left(n, n^{\prime}\right)}\right)$ this replacement is not valid. Therefore, we calculate approximately by using (22), (23) the ratios:

$$
\frac{I_{1}^{\left(n, n^{\prime}-1\right)}}{I_{1}^{\left(n, n^{\prime}\right)}} \approx \frac{3 n^{\prime}}{2\left(n+n^{\prime}\right)}, \quad \frac{I_{1}^{\left(n-1, n^{\prime}\right)}}{I_{1}^{\left(n, n^{\prime}\right)}} \approx \frac{3 n}{n+n^{\prime}} .
$$

Third, we assume, that the leading contribution in neutrino emissivity gives at fixed $n$ by small vicinity of $m=2 n$, because functions $I_{0}^{(n, m)}(v), I_{1}^{(n, m)}(v)$ have delta-like burst in that domain. Hence, we replace $n^{\prime}=2 n$, where it does not cause confusion:

$$
n^{\prime} \frac{n^{\prime}-2 n}{2\left(n+n^{\prime}\right)} \rightarrow \frac{n^{\prime}-2 n}{3}, \quad n \frac{2 n-n^{\prime}}{n+n^{\prime}} \rightarrow \frac{2 n-n^{\prime}}{3} .
$$

After integrations on transverse components and simplifications, the neutrino emissivity (12) could be presented in the forms:

$$
\begin{aligned}
& Q^{(\mathrm{cr})}=\frac{G_{F}^{2} \cos ^{2} \theta_{c} g_{v}^{2}}{16 \pi^{6}}(e B)^{2} \sum_{n, n^{\prime}} \int d^{3} k \int \frac{d p_{z}}{\epsilon} f_{e} \int \frac{d \mathcal{P}_{1 z}}{E_{n}} f_{u} \int \frac{d \mathcal{P}_{2 z}}{E_{n^{\prime}}}\left(1-f_{d}\right) \\
& \cdot \delta_{\|}\left(\mathcal{P}_{1}+p-\mathcal{P}_{2}-k\right)\left(I _ { 0 } ^ { ( n , n ^ { \prime } ) } \left(-(1+c)^{2} \Phi_{\|}+2[k(\tilde{\Lambda}+\tilde{\phi}) p]\left[\left(1+c^{2}\right)\left(\mathcal{P}_{1} \tilde{\Lambda} \mathcal{P}_{2}\right)-\right.\right.\right. \\
&\left.\left.\quad-\left(1-c^{2}\right) m_{u} m_{d}+2 c\left(\mathcal{P}_{1} \tilde{\phi} \mathcal{P}_{2}\right)\right]\right)+ \\
&\left.+v^{2} I_{1}^{\left(n, n^{\prime}\right)} \mathcal{P}_{\perp}^{2}\left((1+c)^{2}\left[\mathcal{P}_{1}(\tilde{\Lambda}+\tilde{\phi}) p\right]+(1-c)^{2}\left[\mathcal{P}_{2}(\tilde{\Lambda}+\tilde{\phi}) p\right]\right)\right),
\end{aligned}
$$

where $\mathcal{P}_{\perp}^{2}=2 e B\left(n^{\prime}-2 n\right) / 3, \delta_{\|}\left(\mathcal{P}_{1}+p-\mathcal{P}_{2}-k\right)=\delta\left(E_{n}+\epsilon-E_{n^{\prime}}-\omega\right) \delta\left(\mathcal{P}_{1 z}+p_{z}-\mathcal{P}_{2 z}-k_{z}\right)$.

\section{Integrals on parallel components}

Firstly, for unification of calculations both processes, we rework artificially by using the conservation of energy and parallel component of momentum in the crossing process:

$$
f_{u} f_{e}\left(1-f_{d}\right)=f_{d}\left(1-f_{u}\right)\left(1-f_{e}\right) \exp \left(-\frac{\omega}{T}\right) \exp \left(\frac{\mu_{e}-{ }_{\Delta} \mu}{T}\right),
$$

where ${ }_{\Delta} \mu \equiv \mu_{d}-\mu_{u}$. As one can see, the expression (28) for neutrino emissivity contain manifestly covariant, from the viewpoint of Lorenz transformations along the magnetic field strength, integrals on parallel components of quarks:

$$
\begin{gathered}
\mathcal{I}_{s} \equiv \int_{-\infty}^{\infty} \frac{d \mathcal{P}_{1 z}}{E_{n}} \int_{-\infty}^{\infty} \frac{d \mathcal{P}_{2 z}}{E_{n^{\prime}}} f_{d}\left(1-f_{u}\right) \delta_{\|}\left(\mathcal{P}_{2}-\mathcal{P}_{1}-q\right), \\
\mathcal{I}_{V}^{(1) \mu} \equiv \int_{-\infty}^{\infty} \frac{d \mathcal{P}_{1 z}}{E_{n}} \int_{-\infty}^{\infty} \frac{d \mathcal{P}_{2 z}}{E_{n^{\prime}}} f_{d}\left(1-f_{u}\right) \delta_{\|}\left(\mathcal{P}_{2}-\mathcal{P}_{1}-q\right) \mathcal{P}_{1 \|}^{\mu}, \\
\mathcal{I}_{V}^{(2) \mu} \equiv \int_{-\infty}^{\infty} \frac{d \mathcal{P}_{1 z}}{E_{n}} \int_{-\infty}^{\infty} \frac{d \mathcal{P}_{2 z}}{E_{n^{\prime}}} f_{d}\left(1-f_{u}\right) \delta_{\|}\left(\mathcal{P}_{2}-\mathcal{P}_{1}-q\right) \mathcal{P}_{2 \|}^{\mu},
\end{gathered}
$$




$$
\begin{gathered}
\mathcal{I}_{T}^{\alpha \beta} \equiv \int_{-\infty}^{\infty} \frac{d \mathcal{P}_{1 z}}{E_{n}} \int_{-\infty}^{\infty} \frac{d \mathcal{P}_{2 z}}{E_{n^{\prime}}} f_{d}\left(1-f_{u}\right) \delta_{\|}\left(\mathcal{P}_{2}-\mathcal{P}_{1}-q\right) \mathcal{P}_{1 \|}^{\alpha} \mathcal{P}_{2 \|}^{\beta}, \\
\mathcal{I}_{\phi} \equiv \int_{-\infty}^{\infty} \frac{d \mathcal{P}_{1 z}}{E_{n}} \int_{-\infty}^{\infty} \frac{d \mathcal{P}_{2 z}}{E_{n^{\prime}}} f_{d}\left(1-f_{u}\right) \delta_{\|}\left(\mathcal{P}_{2}-\mathcal{P}_{1}-q\right)\left(\mathcal{P}_{1} \tilde{\phi} \mathcal{P}_{2}\right)=0,
\end{gathered}
$$

where $\mathcal{P}_{1 \|}^{\alpha}=\left(\mathcal{P}_{1} \tilde{\Lambda}\right)^{\alpha}$, indices $\mu, \alpha, \beta$ runing values of $(0,3), q \equiv p+k$ - for decay and $q \equiv p-k$ - for crossing. As kinematics analysis shows, $q_{0}=\epsilon+\omega>0\left(n^{\prime}-2 n>0\right)$ for decay and $q_{0}=\epsilon-\omega<0$ $\left(n^{\prime}-2 n<0\right)$ for crossing. We note also, that similar integrals over structures like $\left(p_{1} \tilde{\phi} p_{2}\right)$ are zero, while integrals $\mathcal{I}_{V}^{(1) \mu}, \mathcal{I}_{T}^{\alpha \beta}$ can be expressed in terms of $\mathcal{I}_{s}$ :

$$
\begin{gathered}
\mathcal{I}_{v}^{(1) \mu}=\frac{\mathcal{P}_{\perp}^{2}-q_{\|}^{2}}{2 q_{\|}^{2}} \mathcal{I}_{s} q_{\|}^{\mu}, \quad \mathcal{I}_{v}^{(2) \mu}=\frac{\mathcal{P}_{\perp}^{2}+q_{\|}^{2}}{2 q_{\|}^{2}} \mathcal{I}_{s} q_{\|}^{\mu}, \\
\mathcal{I}_{T}^{\alpha \beta}=\left(a \tilde{\Lambda}^{\alpha \beta}+b \frac{q_{\|}^{\alpha} q_{\|}^{\beta}}{q_{\|}^{2}}\right) \mathcal{I}_{s}, \\
a=-\frac{\mathcal{P}_{\perp}^{2}+q_{\|}^{2}}{2}+\frac{2}{3} e B n^{\prime}+m_{d}^{2}-\frac{\left(\mathcal{P}_{\perp}^{2}\right)^{2}-\left(q_{\|}^{2}\right)^{2}}{4 q_{\|}^{2}}, \\
b=-\frac{\left(\mathcal{P}_{\perp}^{2}\right)^{2}-\left(q_{\|}^{2}\right)^{2}}{2 q_{\|}^{2}}+\frac{\mathcal{P}_{\perp}^{2}+q_{\|}^{2}}{2}-\frac{2}{3} e B n^{\prime}-m_{d}^{2},
\end{gathered}
$$

where $q_{\|}^{2}=(q \tilde{\Lambda} q)$.

One can evaluate the scalar integral $\mathcal{I}_{s}$ by using the approximation [10]:

$$
f_{d}\left(1-f_{u}\right) \approx S \cdot \delta\left(E_{n^{\prime}}-\mu_{d}\right),
$$

where the area of overlap of strongly degenerate fermi-distributed functions is:

$$
S=\int_{M_{1}}^{\infty} d E_{n} \int_{M_{2}}^{\infty} d E_{n^{\prime}} f_{d}\left(1-f_{u}\right) \delta\left(E_{n^{\prime}}-E_{n}-q_{0}\right) \approx \frac{\Delta \mu-q_{0}}{1-\exp \left(-\frac{\Delta \mu-q_{0}}{T}\right)},
$$

where $M_{1}=\sqrt{4 e B n / 3+m_{u}^{2}}, M_{2}=\sqrt{2 e B n^{\prime} / 3+m_{d}^{2}}$. By using (39), (40), assuming $\mu_{d} \gg \epsilon, \omega$ and neglecting $m_{u, d}^{2}$ in comparison with $\mu_{d}^{2}, e B n^{\prime}$, one obtains roughly:

$$
\mathcal{I}_{s} \approx \frac{2 \delta\left(\cos \theta-\cos \theta_{1}^{*}\right)+\delta\left(\cos \theta-\cos \theta_{2}^{*}\right)}{\mu_{d}^{2} v_{F}^{2} \omega},
$$

where $v_{F}^{2} \equiv 1-2 e B n^{\prime} / 3 \mu_{d}^{2}$ is Fermi velocity squared for d-quark, $\theta$ is the angle between neutrino momentum $\vec{k}$ and magnetic field strength $\vec{B}$. The explicit form for $\cos \theta_{1}^{*}\left(\cos \theta_{2}^{*}=-\cos \theta_{1}^{*}\right)$ is not required, because, after substitution (29), (35), (36), (41) in neutrino emissivity (28), the integrand does not depend on it. Thus, the integration over $d^{3} k=2 \pi \omega^{2} d \omega d(-\cos \theta)$ gives effectively $4 \pi \omega^{2} d \omega$.

We note, that approximation (39) take place in the rest frame of the quark matter. If the matter moves along the magnetic field, $\delta\left(E_{n^{\prime}}-\mu_{d}\right)$ will be transformed to:

$$
\delta\left(E_{n^{\prime}}-\mu_{d}\right) \rightarrow \frac{\sqrt{q_{\|}^{2}}}{\mu_{d}} \delta\left(\sqrt{q_{\|}^{2}}-\Delta\right)
$$


where $\Delta=\sigma \frac{e B}{3 \mu_{d}}\left(n^{\prime}-2 n\right)$, and $\sigma$ - is sign function of $q_{0}$, or $\left(n^{\prime}-2 n\right)(\sigma>0$ in decay and $\sigma<0$ in crossing). Thus, we have the the constraint on Lorenz-invariant $\sqrt{q_{\|}^{2}} \approx \Delta$. Together with conservation laws of energy and parallel momentum, that constraint determine the region of integration on $\epsilon, \omega-$ variables. We note also, that $\sqrt{q_{\|}^{2}} / \mu_{d}$ is a small number $\left(\sqrt{q_{\|}^{2}} / \mu_{d} \ll 1\right)$.

By assuming, that:

$$
\mu_{d}^{2}, e B n^{\prime} \gg \Delta^{2}, m_{u, d}^{2}, \sigma e B\left(n^{\prime}-2 n\right),
$$

we take the leading contributions in neutrino emissivities:

$$
\begin{aligned}
& Q^{(\mathrm{cr})=} \frac{G_{F}^{2} \cos ^{2} \theta_{c}\left(g_{v}^{2}+g_{a}^{2}\right)}{\pi^{5}}(e B)^{2} e^{\frac{\mu_{e}-\mu^{u}}{T}} \sum_{n, n^{\prime}} \frac{1}{v_{F}^{2}} \int_{\mu_{e}}^{\infty} d \epsilon \int_{\epsilon+\Delta}^{\infty} d \omega \omega e^{-\frac{\omega}{T}} \frac{\mu \mu-\epsilon+\omega}{1-e^{-\frac{\mu^{\mu}-\epsilon+\omega}{T}}} . \\
& \cdot\left(\omega-\epsilon-\sqrt{(\epsilon-\omega)^{2}-\Delta^{2}}\right) I_{0}^{\left(n, n^{\prime}\right)}\left(2+\left[1+v_{F}^{2}\right] \frac{2 \epsilon\left(\omega-\epsilon-\sqrt{(\epsilon-\omega)^{2}-\Delta^{2}}\right)}{\Delta^{2}}\right), \\
& Q^{(\mathrm{dec})}=\frac{G_{F}^{2} \cos ^{2} \theta_{c}\left(g_{v}^{2}+g_{a}^{2}\right)}{\pi^{5}}(e B)^{2} \sum_{n, n^{\prime}} \frac{1}{v_{F}^{2}} \int_{\mu_{e}}^{\infty} d \epsilon \int_{\Delta^{2} /(2 \epsilon)}^{\infty} d \omega \omega \frac{(\epsilon+\omega-\Delta \mu)}{e^{\frac{\epsilon+\omega-\mu^{\mu}}{T}}-1} . \\
& \cdot\left(\omega+\epsilon-\sqrt{(\epsilon+\omega)^{2}-\Delta^{2}}\right) I_{0}^{\left(n, n^{\prime}\right)}\left(2+\left[1+v_{F}^{2}\right] \frac{2 \epsilon\left(\omega+\epsilon-\sqrt{(\epsilon+\omega)^{2}-\Delta^{2}}\right)}{\Delta^{2}}\right) .
\end{aligned}
$$

In evaluating the rest of the integrals (44), (45) over $(\epsilon, \omega)$ - variables we assume, that the deviation from beta-equilibrium $\mu_{e}-{ }_{\Delta} \mu$ is positive and small enough number $\left(\mu_{e}-{ }_{\Delta} \mu>0, \mu_{e}-{ }_{\Delta} \mu \ll \mu_{e}\right)$, while $\left(\mu_{e}-{ }_{\Delta} \mu\right) / T \gg 1$. As a consequence of exponentially suppressed integrands, the leading contributions in integrals gives the regions $\epsilon-\mu_{e} \sim T, \omega-\mu_{e}-\Delta \sim T$ (crossing) and $\epsilon-\mu_{e} \sim T, \omega-\Delta^{2} /\left(2 \mu_{e}\right) \sim T$ (decay). Thus, the leading contributions in neutrino emissivities could be presented in the form:

$$
\begin{gathered}
Q^{(\mathrm{cr})}=\frac{2 G_{F}^{2} \cos ^{2} \theta_{c}\left(g_{v}^{2}+g_{a}^{2}\right)}{\pi^{5}}(e B)^{2} \Delta \mu \mu_{e}^{2} T^{2} e^{-\frac{\mu^{\mu}}{T}} \sum_{n, n^{\prime}} \frac{1+v_{F}^{2}}{v_{F}^{2}} e^{-\frac{\Delta}{T}} I_{0}^{\left(n, n^{\prime}\right)}, \\
Q^{(\mathrm{dec})}=\frac{G_{F}^{2} \cos ^{2} \theta_{c}\left(g_{v}^{2}+g_{a}^{2}\right)}{4 \pi^{5}}(e B)^{2} \frac{\left(\mu_{e}-\Delta \mu\right)}{\mu_{e}^{2}} T^{2} e^{-\frac{\mu_{e}-\Delta^{\mu}}{T}} \sum_{n, n^{\prime}} \frac{1-v_{F}^{2}}{v_{F}^{2}} \Delta^{4} e^{-\frac{\Delta^{2}}{2 \mu_{e} T}} I_{0}^{\left(n, n^{\prime}\right)} .
\end{gathered}
$$

\section{Summations over $n, n^{\prime}$}

We introduce new variables $k=2 n-n^{\prime}, k=n^{\prime}-2 n$ for crossing and decay accordingly, and replace sums:

$$
\sum_{n, n^{\prime}} \rightarrow \sum_{n=1}^{N_{1}} \sum_{k=1}^{2 n}, \quad \sum_{n, n^{\prime}} \rightarrow \sum_{n^{\prime}=0}^{N_{2}} \sum_{k=1}^{n^{\prime}}
$$

where $N_{1}=3 \mu_{u}^{2} / 4 e B, N_{2}=3 \mu_{d}^{2} / 2 e B$.

Next, we express for crossing $n^{\prime}=2 n-k$ in $I_{0}$ and $v_{F}^{2}$. We take only the first component of sum of $k$ because of suppressing exponent, and obtain:

$$
Q^{(\mathrm{cr})} \approx \frac{2 G_{F}^{2} \cos ^{2} \theta_{c}\left(g_{v}^{2}+g_{a}^{2}\right)}{(\pi)^{5}}(e B)^{2} \mu_{e}^{2} \mu T^{2} \exp \left(-\frac{\mu}{T}\right) \exp \left(-\frac{e B}{3 \mu_{d} T}\right) \sum_{n} I_{0}^{(n, 2 n-1)} \frac{\left(1+v_{F}^{2}\right)}{v_{F}^{2}}
$$


where $v_{F}^{2}=1-2 e B(2 n-1) / 3 \mu_{d}^{2}$.

We express in decay process $n=\left(n^{\prime}-k\right) / 2$ and neglect in $I_{0}^{\left(n, n^{\prime}\right)}$ dependence of $k$. This action is giving too high values of $I_{0}^{\left(n, n^{\prime}\right)}$ by summing over $n^{\prime}$. Hence, we obtain the upper bound on neutrino emissivity of decay. We use roughly the Euler-Macloren formula to sum the rest terms on $k$. In that case, the upper bound on neutrino emissivity of decay process is:

$$
\begin{aligned}
Q^{(\mathrm{dec})} \leqslant \frac{9 \sqrt{\pi} G_{F}^{2} \cos ^{2} \theta_{c}\left(g_{v}^{2}+g_{a}^{2}\right)}{4 \sqrt{2}(\pi)^{5}}(e B) \mu_{d} \sqrt{\mu_{e}}\left(\mu_{e}-{ }_{\Delta} \mu+\frac{5}{2} T\right) T^{\frac{9}{2}} & \\
& \cdot \exp \left(-\frac{\mu_{e}-\Delta \mu}{T}\right) \sum_{n^{\prime}} I_{0}^{\left(\frac{n^{\prime}}{2}, n^{\prime}\right)} \frac{1-v_{F}^{2}}{v_{F}^{2}},
\end{aligned}
$$

where $v_{F}^{2}=1-2 e B n^{\prime} / 3 \mu_{d}^{2}$.

\section{Conclusions}

We research the covariant method of evaluation of neutrino emissivity in direct URCA-processes. In our model the superdense strong magnetized quark medium emits neutrinos. In evaluations, the values of magnetic field corresponds to the case, where the quarks take a lot of Landau-levels, and the electrons take ground Landau-level. In this case the analytically dependence neutrino emissivity of task parameters (chemical potentials quarks and electrons, temperature, magnetic field) is obtained for the first time. In using approximations

$$
\begin{aligned}
& \mu_{u}^{2}, \mu_{d}^{2} \gg m_{q}^{2}(\mu)>e B \gtrsim \mu_{e}^{2} \gg T^{2}, \\
& \mu_{d}^{2}, e B n^{\prime} \gg \Delta^{2}, m_{u, d}^{2}, \sigma e B\left(n^{\prime}-2 n\right),
\end{aligned}
$$

the main result is an exponentially suppression of both processes emissivities. As one can see from formulae (49), (50), in beta-equilibrium the decay goes on and the crossing is suppressed. Without beta-equilibrium both processes are suppressed. Thus, for our medium the stable state is the state with small deflection from beta-equilibrium and forbidden direct URCA-processes.

The decay suppression is a consequence of very small area $S$ (40) of strongly degenerate fermidistributed functions $f_{d}\left(1-f_{u}\right)$ overlap.

The crossing suppression is a consequence of one-dimensional kinematics, which gives the restriction $\omega>\epsilon$, while $\epsilon \geqslant \mu_{e}$.

The main question for further investigation is how do URCA-processes go on at gradual decrease of magnetic field?

The paper is done within "YSU Initiative Scientific Researches" (Project AAAA-A16116070610023-3).

\section{References}

[1] Ozel F., Freire P. Masses, radii, and equation of state of neutron star. Ann. Rev. Astron. Astrophys., 54, 401-440, (2016). 
[2] Weber F., Contera G. A., et al. Properties of high-density matter in neutron stars. Mod. Phys Lett. A, 29, 1430022, (2014).

[3] Potekhin A. Y., Pons J. A., Page D. Neutron stars - cooling and transport. Space. Sci. Rev. 191, 239, (2015).

[4] Turolla R., Zane S., Watts A. Magnetars: the physics behind observations. Rep. Prog. Phys. 78, 116901, (2015).

[5] Iwamoto N. Quark Beta Decay and the Cooling of Neutron Stars. Phys.Rev.Lett., 44, 1637, (1980).

[6] Schmitt A. Dense matter in compact stars. e-Print Arxiv: astro-ph.SR 1001.3294v2., (2010).

[7] Le Bellac M. Thermal Field Theory. (Cambridge University Press, Cambridge, 1996) 269.

[8] Gvozdev A. A., Osokina E. V. Neutrino processes in an external magnetic field in the density matrix formalism, TMP, 170:3, pp 354 - 375, (2012).

[9] Gradshteyn I. S., Ryzhik I. M. Table of Integrals, Series, and Products (7ed). (Academic Press, Oxford, 2007) 1161.

[10] Mikheev N. V., Narynskaya E. N. Neutrino-electron processes in a dense magnetized plasma. Modern Physics Letters A., 15:25, 1551-1556, (2000). 